\title{
The dipeptidyl peptidase-4 inhibitor teneligliptin improved endothelial dysfunction and insulin resistance in the SHR/NDmcr-cp rat model of metabolic syndrome
}

\author{
Hironori Nakagami ${ }^{1}$, Zhengda Pang ${ }^{2}$, Takashi Shimosato ${ }^{3}$, Toshinori Moritani ${ }^{3}$, Hitomi Kurinami ${ }^{1}$, \\ Hiroshi Koriyama ${ }^{1}$, Akiko Tenma ${ }^{1}$, Munehisa Shimamura ${ }^{1}$ and Ryuichi Morishita ${ }^{4}$
}

Diabetes mellitus, hypertension and metabolic syndrome are major risk factors for the occurrence of cardiovascular events. In this study, we used spontaneous hypertensive rat (SHR)/NDmcr-cp (cp/cp) (SHRcp) rats as a model for metabolic syndrome to examine the effects of dipeptidyl peptidase (DPP)-4 inhibition on hypertension, glucose metabolism and endothelial dysfunction. First, we confirmed that SHRcp rats showed very severe obesity, hypertension and endothelial dysfunction phenotypes from 14 to 54 weeks of age. Next, we examined whether the DPP-4 inhibitor teneligliptin $\left(10 \mathrm{mg} \mathrm{kg}^{-1}\right.$ per day per os for 12 weeks) could modify any of these phenotypes. Treatment with teneligliptin significantly improved hyperglycemia and insulin resistance, as evidenced by an oral glucose tolerance test and homeostasis model assessment for insulin resistance, respectively. Teneligliptin showed no effects on systolic blood pressure or heart rate. In regard to endothelial function, the vasodilator response to acetylcholine was significantly impaired in SHRcp rats when compared with WKY rats. Long-term treatment with teneligliptin significantly attenuated endothelial dysfunction through the upregulation of endothelium-derived nitric oxide synthase mRNA. These results demonstrate that long-term treatment with teneligliptin significantly improved endothelial dysfunction and glucose metabolism in a rat model of metabolic syndrome, suggesting that teneligliptin treatment might be beneficial for patients with hypertension and/or diabetes.

Hypertension Research (2014) 37, 629-635; doi:10.1038/hr.2014.53; published online 13 March 2014

Keywords: DPP-4 inhibitor; endothelial function; insulin resistance; SHRcP

\section{INTRODUCTION}

Although hypertension is a well-known risk factor for cardiovascular disease, obesity is another risk factor that has reached epidemic proportions in the developed world as the number of overweight or obese people continues to increase. Obesity is associated with an increased risk of diabetes, dyslipidemia and hypertension. Together, these conditions are known as metabolic syndrome ${ }^{1,2}$ and are strong risk factors for the development of cardiovascular disease. Furthermore, a growing body of evidence has recently demonstrated that endothelial function is impaired in these conditions, particularly in patients with type 2 diabetes mellitus. ${ }^{3}$

Although several mechanisms have been shown to be involved in the endothelial dysfunction observed in hypertension and diabetes, the reduced bioavailability of endothelium-derived nitric oxide has become a topic of particular interest. ${ }^{4}$ Furthermore, the delivery of insulin to skeletal muscle and the insulin-dependent uptake of glucose by skeletal muscle are impaired in patients with type 2 diabetes. 5,6 Insulin resistance is also a risk factor for atherosclerosis, and patients with insulin resistance have impaired endothelial function. Recently, Kubota et al. ${ }^{7}$ demonstrated that endothelial insulin signaling mediates insulin-stimulated capillary recruitment and increases interstitial insulin levels, which consequently facilitates the uptake of glucose by skeletal muscle. These authors suggested that impaired insulin signaling in endothelial cells, with a concomitant reduction of insulin-related substrate 2 expression and decrease of insulin-induced endothelium-derived nitric oxide synthase (eNOS) phosphorylation,

\footnotetext{
${ }^{1}$ Division of Vascular Medicine and Epigenetics, Osaka University United Graduate School of Child Development, Osaka University, Kanazawa University, Hamamatsu University School of Medicine, Chiba University and University of Fukui, Osaka, Japan; ${ }^{2}$ Department of Geriatric Medicine, Graduate School of Medicine, Osaka University, Osaka, Japan; ${ }^{3}$ Research Department, NISSEI BILIS Co. Ltd., Shiga, Japan and ${ }^{4}$ Department of Clinical Gene Therapy, Graduate School of Medicine, Osaka University, Osaka, Japan Correspondence: Professor H Nakagami, Division of Vascular Medicine and Epigenetics, Osaka University Graduate School of Child Development, Osaka University, Kanazawa University and Hamamatsu University School of Medicine, 2-1 Yamada-oka Suita, Suita 565-0871, Japan.

E-mail: nakagami@cgt.med.osaka-u.ac.jp

or Professor R Morishita, Department of Clinical Gene Therapy, Graduate School of Medicine Osaka University, 2-2 Yamada-oka Suita, Suita 565-0871, Japan. E-mail: morishit@cgt.med.osaka-u.ac.jp

Received 2 December 2013; revised 23 December 2013; accepted 25 December 2013; published online 13 March 2014
} 
reduces insulin-induced glucose uptake by skeletal muscle, at least in part through decreased capillary recruitment and lower interstitial insulin levels in the skeletal muscle. These results suggest that insulin resistance in skeletal muscle may be due to impaired insulin signaling not only in myocytes but also in endothelial cells.

Dipeptidyl peptidase (DPP)-4 inhibitors are potent drugs used to treat patients with type 2 diabetes mellitus. Inhibition of DPP-4 increases levels of biologically intact glucagon-like peptide-1, which enhances glucose metabolism through the upregulation of insulin secretion and the suppression of glucagon release. ${ }^{8,9}$ In this study, we used spontaneous hypertensive rats (SHR)/NDmcr-cp ( $\mathrm{cp} / \mathrm{cp}$ ) (SHRcp rats) as a model for severe metabolic syndrome involving both hypertension and obesity. SHRcp rats are a congenic SHR strain with a nonsense mutation in the leptin receptor, ${ }^{10}$ and these animals exhibit obesity, hypertension, insulin resistance, glucose intolerance and hyperlipidemia. ${ }^{11-13}$ Using this SHRcp rat model, we examined the effects of the DPP-4 inhibitor teneligliptin on endothelial dysfunction and glucose metabolism.

\section{METHODS}

\section{Animals}

This study was approved by the Institute of Experimental Animal Science Committee at Osaka University Medical School and was carried out in accordance with the institutional guidelines. Experiments were performed in WKY/Izm and SHR/NDmcr-cp (SHRcp) rats (12 weeks of age) from Japan SLC (Hamamatsu, Japan). Teneligliptin was obtained from Mitsubishi Tanabe Pharma (Osaka, Japan). SHRcp rats were administered $10 \mathrm{mg} \mathrm{kg}^{-1}$ per day teneligliptin by oral gavage for 12 weeks (from 12 to 24 weeks of age). The rats were given free access to food and water during the course of the experiments.

Measurements of body weight, tissue weight, blood pressure and heart rate

The body weight of each rat was measured weekly. At the end of the study, the weights of the heart, liver, pancreas, kidney, and epididymal, mesenteric and retroperitoneal adipose tissue were determined. Systolic blood pressure was measured at 14, 24, 34 and 54 weeks of age using the tail-cuff method (MK2000, Muromachi Kikai, Tokyo, Japan). Fasting blood samples were also collected at 14, 24, 34 and 54 weeks of age.

a
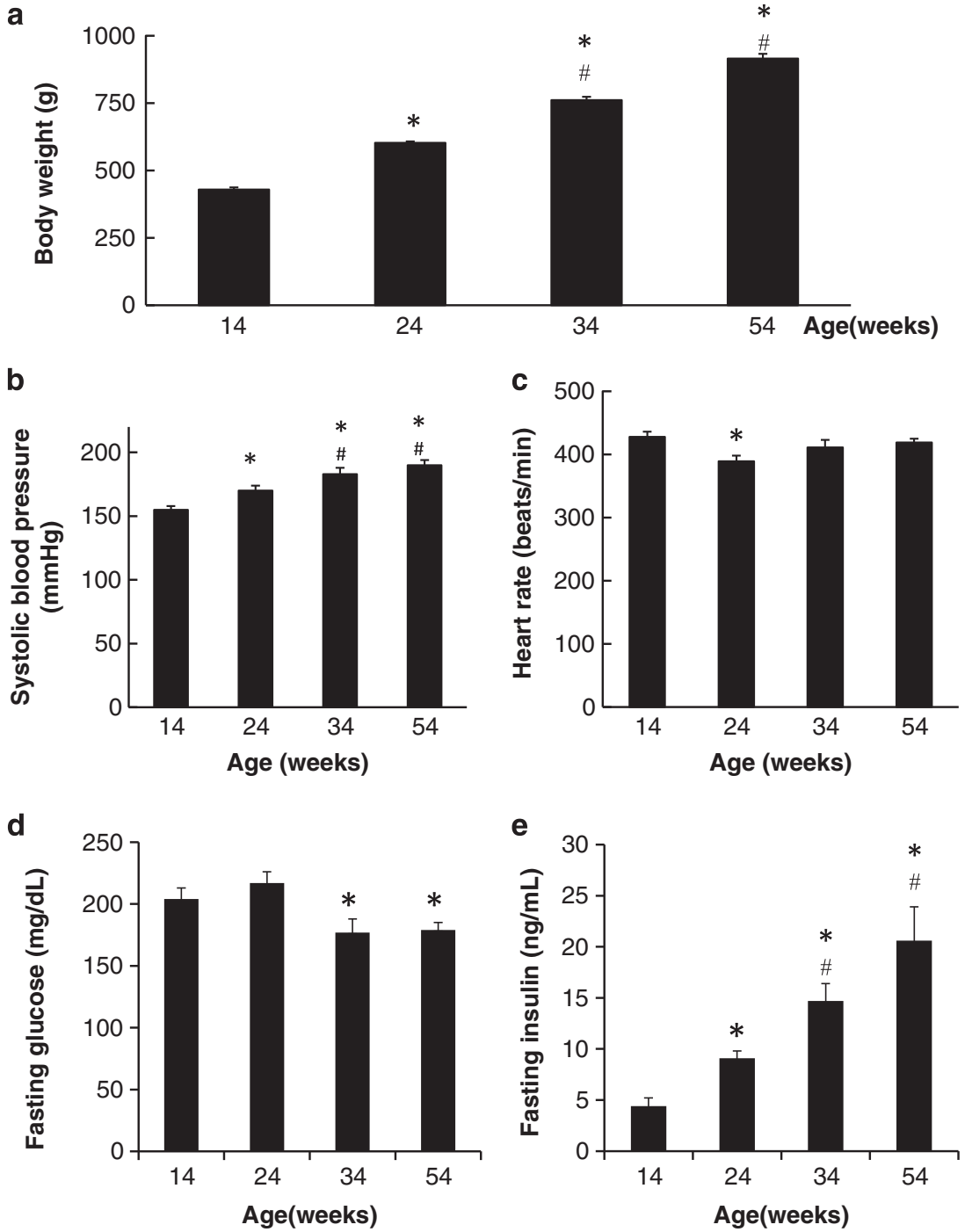

Figure 1 Body weight, systolic blood pressure, heart rate, fasting glucose levels and insulin levels in SHR/NDmcr-cp (cp/cp) (SHRcp) rats at 14,24 , 34 and 54 weeks of age. (a) Body weight (g). (b) Systolic blood pressure ( $\mathrm{mm} \mathrm{Hg}$ ). (c) Heart rate (beats per min). (d) Fasting glucose levels (mg dl-1). (e) Fasting insulin levels $(\mathrm{ng} \mathrm{ml})^{-1}$ ). $N=10$ for each group. ${ }^{*} P<0.05$ vs. 14-week-old rats, ${ }^{\#} P<0.05$ vs. 24-week-old rats. 


\section{Assessment of endothelial dysfunction}

We measured endothelial function by evaluating the vasodilator response to acetylcholine (Ach) ${ }^{14}$ In brief, freshly isolated carotid, thoracic and mesenteric arteries were cut into $3 \mathrm{~mm}$ rings and mounted in $10 \mathrm{ml}$ organ baths containing the Krebs-Henseleit buffer $\left(120 \mathrm{mmoll}^{-1} \mathrm{NaCl}, 4.7 \mathrm{mmoll}^{-1}\right.$ $\mathrm{KCl}, 2.5 \mathrm{mmoll}^{-1} \mathrm{CaCl}_{2}, 1.2 \mathrm{mmoll}^{-1} \mathrm{MgSO}_{4}, 1.2 \mathrm{mmoll}^{-1} \mathrm{KH}_{2} \mathrm{PO}_{4}$, $25 \mathrm{mmoll}^{-1} \mathrm{NaHCO}_{3}$ and $5.5 \mathrm{mmoll}^{-1}$ glucose, $\mathrm{pH}$ 7.4). The samples were maintained at $37^{\circ} \mathrm{C}$ and aerated with $95 \% \mathrm{O}_{2}$ and $5 \% \mathrm{CO}_{2}$. The aortic rings were equilibrated for $60 \mathrm{~min}$ and the bathing fluid was changed every $15 \mathrm{~min}$. Isometric tension studies were performed using a transducer (TB-611T and AP-621G, Nihon Koden, Tokyo, Japan). Optimal resting tension was determined from baseline studies, and then the responses to vasoactive drugs were determined. Cumulative dose-response curves for Ach $\left(10^{-9}-10^{-5} \mathrm{moll}^{-1}\right)$ were established after the aortic rings were submaximally precontracted with phenylephrine $\left(3 \times 10^{-6} \mathrm{moll}^{-1}\right)$. Finally, the maximal relaxant response to papaverine $\left(10^{-4} \mathrm{moll}^{-1}\right)$ was recorded. The percent relaxation at several different doses of $\mathrm{ACh}$ and single nucleotide polymorphism was calculated relative to the maximum relaxation (that is, the response to papaverine). ACh was obtained from Daichi Fine Chemical (Toyama, Japan), and all other chemicals were obtained from Sigma (St Louis, MO, USA).

\section{Real-time reverse transcription-PCR analysis}

The mRNA expression of rat endothelial nitric oxide synthase (eNOS), tumor necrosis factor (TNF)- $\alpha$ and intercellular adhesion molecule-1 (ICAM-1) was measured using real-time reverse transcription-PCR (RT-PCR). Total RNA was extracted from tissue samples using the ISOGEN reagent (NIPPON GENE, Toyama, Japan). Complementary DNA was synthesized using the Thermo Script RT-PCR System (Invitrogen, Carlsbad, CA, USA). Relative gene copy numbers for eNOS, TNF- $\alpha$, ICAM- 1 and glyceraldehyde-3-phosphate dehydrogenase were determined by real-time RT-PCR using TaqMan Gene Expression Assays (eNOS: Rn02132634_s1, TNF- $\alpha$ : Rn01525859_g1, ICAM1: Rn00564227_m1 and glyceraldehyde-3-phosphate dehydrogenase: Rn01775763_g1). Absolute gene copy numbers were normalized to glyceraldehyde-3-phosphate dehydrogenase using a standard curve.

\section{Glucose tolerance test}

The rats were fasted for $16 \mathrm{~h}$. At the end of the fast, the rats were orally administered glucose $\left(2.0 \mathrm{mgg}^{-1}\right.$ body weight). Blood samples were collected from the tail vein at different time intervals, and glucose levels $\left(\mathrm{mg} \mathrm{dl}^{-1}\right)$ were determined using the glucose oxidase method with an Antosense device (Horiba, Kyoto, Japan). Whole-blood samples were collected and centrifuged in heparinized tubes, and the plasma was stored at $-20^{\circ} \mathrm{C}$. Insulin levels were determined using an insulin enzyme-linked immunosorbent assay kit (MORINAGA, Yokohama, Japan). Insulin sensitizing effects were evaluated using the homeostasis model assessment for insulin resistance (fasting plasma insulin $\left(\mu \mathrm{Uml}^{-1}\right) \times$ fasting plasma glucose $\left.\left(\mathrm{mg} \mathrm{dl}^{-1}\right) / 405\right)$.

\section{Statistical analysis}

Statistical analyses were performed using the Stat-View 5.0 software program (SAS Institute, Cary, NC, USA). Data were compared using $t$-tests between two groups. A $P$-value of less than 0.05 was considered statistically significant.

\section{RESULTS}

\section{SHRcp phenotypes are a novel model of aging}

We first analyzed the progression of SHRcp phenotypes in the rats at $14,24,34$ and 54 weeks of age. Body weight continuously increased as the rats aged (Figure 1a). Tissue weights (heart, kidney, pancreas, liver and fat) also increased with age, and retroperitoneal fat showed the greatest increase in weight over time (Table 1). Systolic blood pressure also increased with age; however, the heart rate did not significantly change (Figures $1 \mathrm{~b}$ and $\mathrm{c}$ ). Unexpectedly, we observed that fasting glucose levels were high at 14 and 24 weeks of age but relatively low at 34 and 54 weeks of age. Fasting insulin levels showed the opposite trend and increased with age. These results indicate that obesity, high
Table 1 Organ weight in SHRcp with aging

\begin{tabular}{lrccc}
\hline & $14 W$ & $24 W$ & $34 W$ & $54 W$ \\
\hline Heart (g) & $1.30 \pm 0.03$ & $1.63 \pm 0.03^{*}$ & $1.71 \pm 0.07^{*}$ & $1.82 \pm 0.03^{*, \dagger}$ \\
Kidney (g) & $1.20 \pm 0.02$ & $1.35 \pm 0.02^{*}$ & $1.50 \pm 0.03^{*, \dagger}$ & $1.68 \pm 0.05^{*, \dagger, \#}$ \\
Pancreas (g) & $0.76 \pm 0.04$ & $1.09 \pm 0.03^{*}$ & $1.25 \pm 0.06^{*}$ & $1.45 \pm 0.09^{*, \dagger, \#}$ \\
Liver (g) & $15.82 \pm 0.33$ & $19.95 \pm 0.41^{*}$ & $22.28 \pm 0.36^{*, \dagger}$ & $20.75 \pm 0.74^{*, \#}$ \\
& & & & \\
Fat & & & & \\
$\quad$ Epididymal (g) & $9.21 \pm 0.16$ & $8.79 \pm 0.16^{\dagger}$ & $8.83 \pm 0.36$ & $6.46 \pm 0.15^{*, \dagger, \#}$ \\
$\quad$ Retroperitoneal (g) & $17.67 \pm 0.57$ & $44.74 \pm 0.88^{*}$ & $66.59 \pm 3.21^{* \dagger}$ & $79.93 \pm 4.08^{*, \dagger, \#}$ \\
$\quad$ Mesenteric (g) & $10.30 \pm 0.25$ & $10.61 \pm 0.47$ & $12.30 \pm 0.56^{* \dagger}$ & $11.12 \pm 0.51$
\end{tabular}

Abbreviations: SHR, spontaneous hypertensive rat; SHRcp, SHR/NDmcr-cp (cp/cp) ${ }^{\star} P<0.05$ vs. $14 \mathrm{~W},{ }^{\dagger} P<0.05$ vs. $24 \mathrm{~W},{ }^{\#} P<0.05$ vs. $34 \mathrm{~W}$.
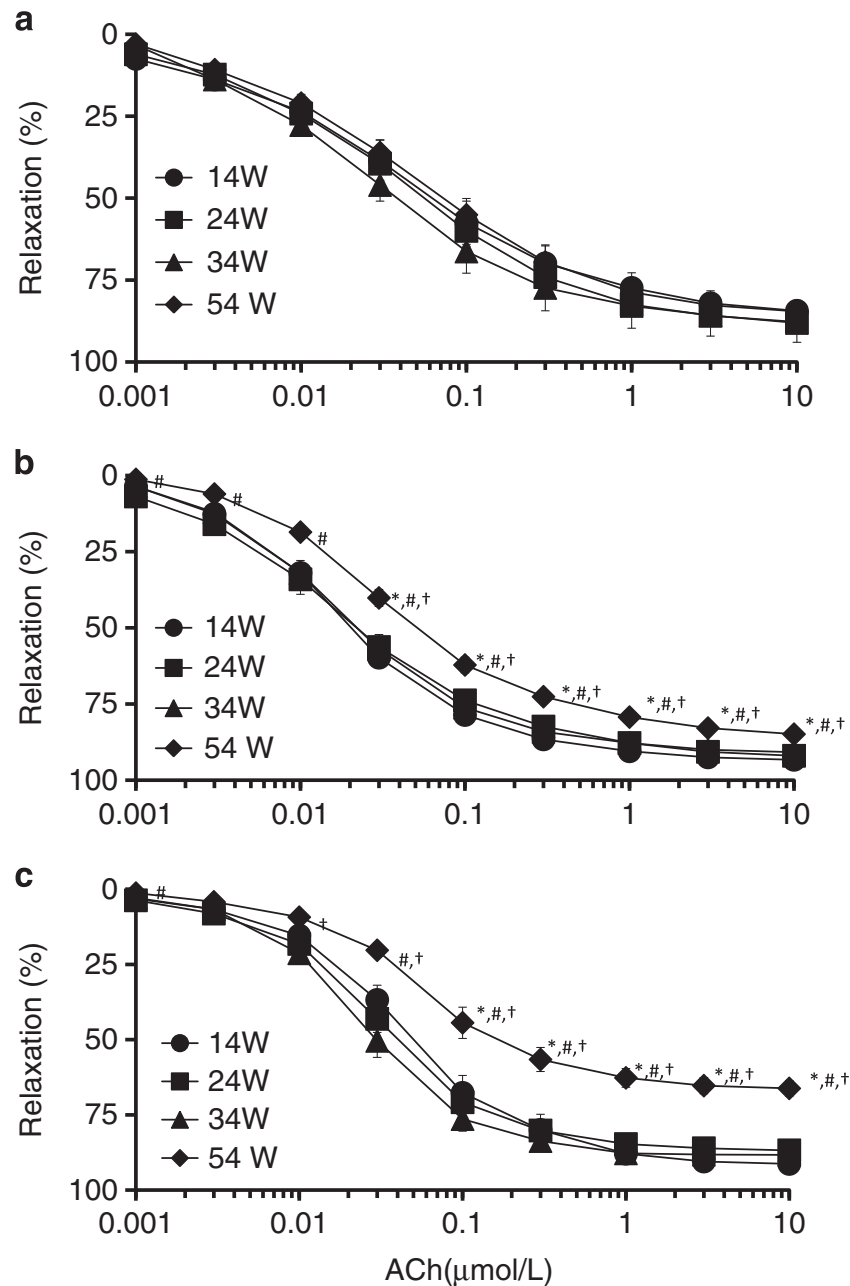

Figure 2 Endothelial function in spontaneous hypertensive rat (SHR)/ NDmcr-cp (cp/cp) (SHRcp) rats at 14, 24, 34 and 54 weeks of age. Cumulative concentration-response curves for acetylcholine (Ach) treatment in isolated carotid (a), thoracic (b) and mesenteric (c) arterial rings. Relaxation rates were calculated as a percentage of the maximum relaxation rate induced by papaverine. ${ }^{*} P<0.05$ vs. 14 -week-old rats, ${ }^{\#} P<0.05$ vs. 24-week-old rats, ${ }^{\dagger} P<0.05$ vs. 34-week-old rats.

blood pressure and insulin levels gradually increased with age in SHRcp rats, whereas fasting glucose levels decreased between 24 and 34 weeks of age through an unknown mechanism. Therefore, we carried out subsequent experiments in rats younger than 24 weeks of age. 

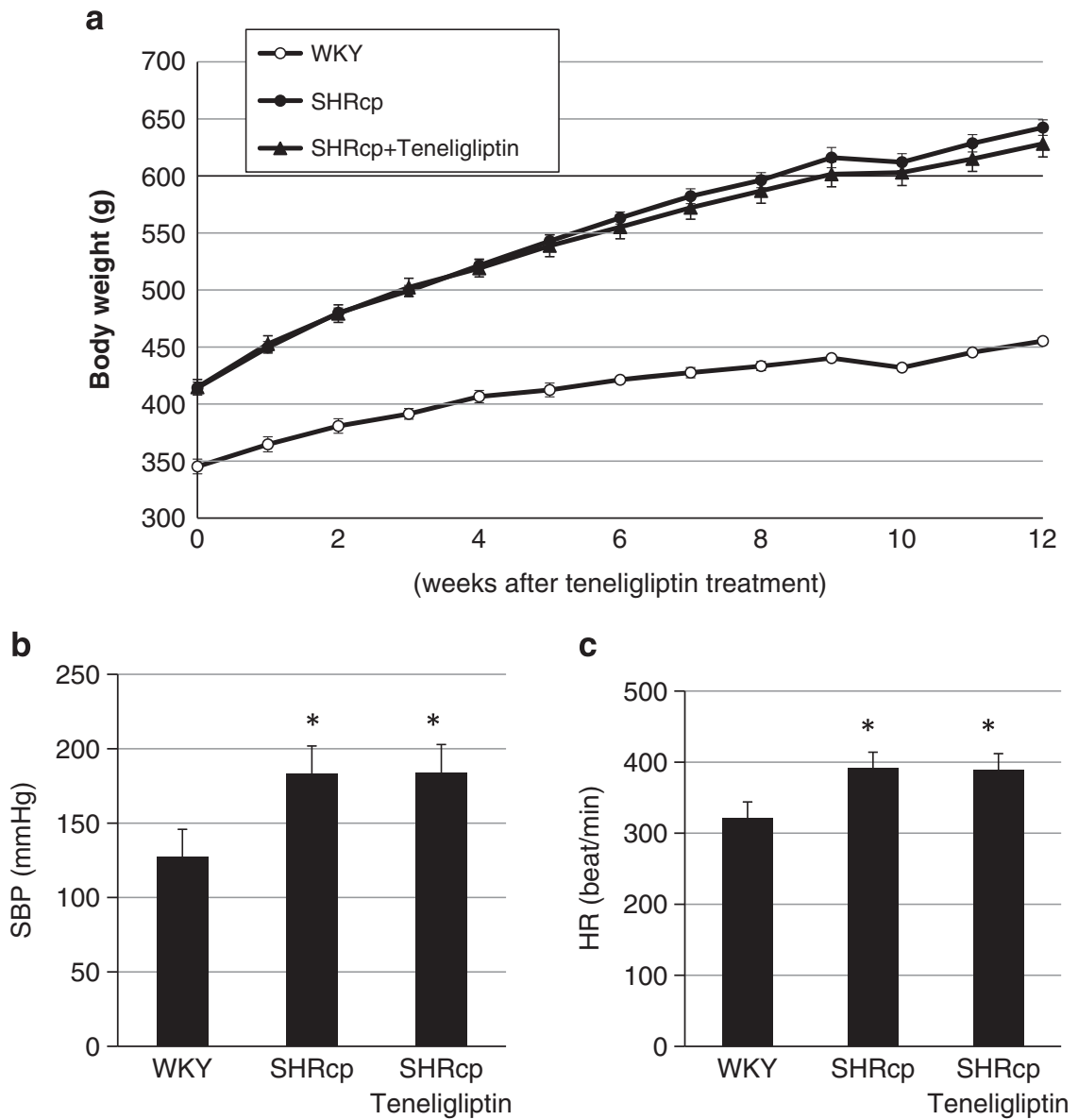

Figure 3 Body weight, systolic blood pressure and heart rate in WKY and SHR/NDmcr-cp (cp/cp) (SHRcp) rats with or without teneligliptin treatment. Body weight was measured weekly from 12 to 24 weeks of age (a) Systolic blood pressure (SBP) (b) and heart rate (HR) (c) were measured at 24 weeks of age. $N=3,6$ and 8 for each group, respectively. ${ }^{*} P<0.05$ vs. WKY rats.

Table 2 Organ weight in SHRcp with teneligliptin treatment

\begin{tabular}{lccc}
\hline & WKY & SHRcp & SHRcp + teneligliptin \\
\hline Heart (g) & $1.22 \pm 0.01$ & $1.64 \pm 0.05^{*}$ & $1.70 \pm 0.04^{*}$ \\
Kidney (g) & $2.60 \pm 0.04$ & $2.73 \pm 0.06$ & $2.84 \pm 0.07$ \\
Pancreas (g) & $1.47 \pm 0.09$ & $0.88 \pm 0.10^{*}$ & $0.78 \pm 0.09^{*}$ \\
Liver (g) & $9.93 \pm 0.07$ & $21.24 \pm 0.52^{*}$ & $22.64 \pm 1.61^{*}$ \\
& & & \\
Fat & & & \\
$\quad$ Epididymal (g) & $7.30 \pm 0.08$ & $7.61 \pm 0.44$ & $8.21 \pm 0.25^{*}$ \\
$\quad$ Retroperitoneal (g) & $8.42 \pm 1.14$ & $39.28 \pm 1.40^{*}$ & $38.01 \pm 0.85^{*}$ \\
Mesenteric (g) & $3.66 \pm 0.37$ & $9.00 \pm 0.39^{*}$ & $9.79 \pm 0.71^{*}$ \\
\hline
\end{tabular}

Abbreviations: SHR, spontaneous hypertensive rat; SHRcp, SHR/NDmcr-cp (cp/cp). ${ }^{*} P<0.05$ vs. WKY.

Next, we evaluated endothelial function by assessing the vasodilator response to Ach. We observed no significant differences in the vasodilator response among samples from rats aged 14, 24 and 34 weeks using three different types of arterial tissues, including the carotid artery (Figure 2a), thoracic artery (Figure 2b) and mesenteric artery (Figure 2c). However, the vasodilator response to Ach was significantly impaired in the thoracic and mesenteric artery samples from the 54-week-old rats (Figures $2 \mathrm{~b}$ and c). Although the mesenteric artery is more sensitive than other arteries in terms of endothelial function, it appears that endothelial function is preserved until at least 34 weeks of age in SHRcp rats.

\section{Teneligliptin treatment in SHRcp rats}

SHRcp rats were treated with teneligliptin $\left(10 \mathrm{mg} \mathrm{kg}^{-1}\right.$ per day per os for 12 weeks) beginning at 12 weeks of age. With respect to body weight, SHRcp rats were considerably heavier than WKY rats. Body weight in the teneligliptin-treated group was slightly decreased when compared with the nontreated SHRcp group (Figure 3a). The heart, liver, retroperitoneal fat and mesenteric fat from SHRcp rats were significantly heavier than those from WKY rats, and treatment with teneligliptin did not affect the weight of these tissues (Table 2). Independent of teneligliptin treatment, the weight of the pancreas was lower in SHRcp rats when compared with WKY rats. We also measured systolic blood pressure and the heart rate at 24 weeks of age. We found that the SHRcp rats had higher blood pressure and faster heart rates when compared with the WKY rats, and treatment with teneligliptin did not affect these metrics (Figures $3 b$ and $c$ ).

The effects of teneligliptin on glucose metabolism and endothelial dysfunction in SHRcp rats

We further examined the effects of teneligliptin on glucose metabolism in SHRcp rats using an oral glucose tolerance test. As expected, hyperglycemia was observed in the SHRcp rats but not in the WKY animals. Importantly, treatment with teneligliptin caused a significant 
a

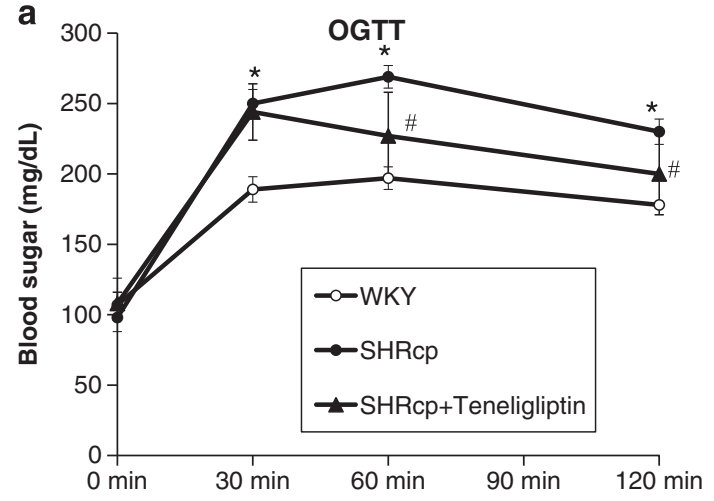

b

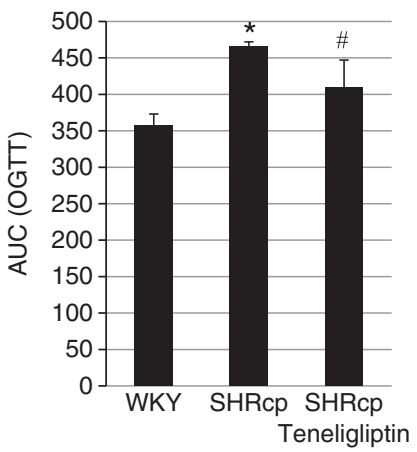

C

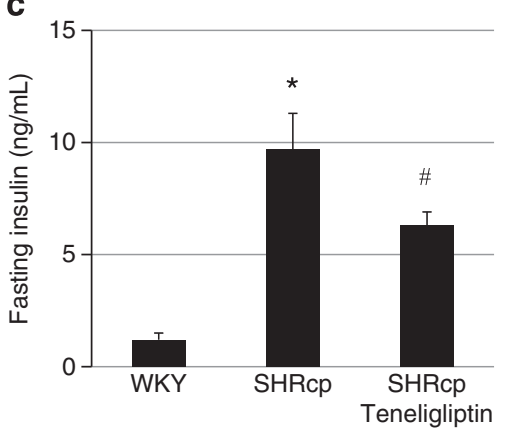

d

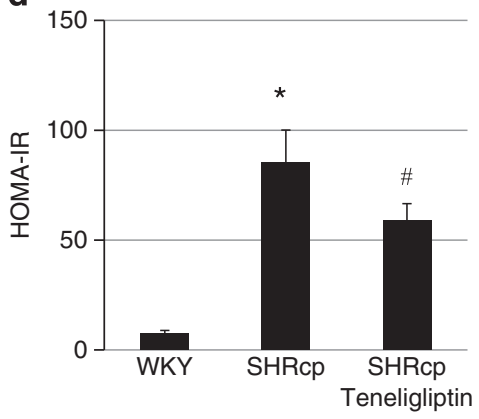

Figure 4 Glucose metabolism in WKY and SHR/NDmcr-cp (cp/cp) (SHRcp) rats with or without teneligliptin treatment. (a) Blood sugar levels at 0, 30,60 and $120 \mathrm{~min}$ after glucose administration as assessed by an oral glucose tolerance test (OGTT). (b) The area under the curve (AUC) of the OGTT curve. (c) Fasting insulin levels $\left(\mathrm{ng} \mathrm{ml}^{-1}\right)$. (d) homeostasis model assessment for insulin resistance was calculated using the fasting glucose and insulin levels, as described in the Methods section. $N=3,6$ and 8 for each group, respectively. ${ }^{*} P<0.05$ vs. WKY rats, ${ }^{\#} P<0.05$ vs. SHRcp.

decrease in blood sugar 60 and 120 min after glucose administration (Figures $4 \mathrm{a}$ and $\mathrm{b}$ ). Fasting insulin levels and homeostasis model assessment for insulin resistance were significantly increased in the SHRcp rats when compared with the WKY rats; furthermore, this effect was mitigated by treatment with teneligliptin (Figures $4 \mathrm{c}$ and $\mathrm{d}$ ).

We next chose to focus on endothelial function in this system by using the vasodilator response to Ach. Endothelial function in the mesenteric artery was significantly impaired in SHRcp rats when compared with WKY rats, and we observed that treatment with teneligliptin partially alleviated this dysfunction (Figures $5 \mathrm{a}$ and $\mathrm{b}$ ). We quantified mRNA levels using real-time RT-PCR and found that eNOS expression was significantly decreased in SHRcp rats when compared with WKY rats; furthermore, teneligliptin treatment significantly increased eNOS expression in the SHRcp rats (Figure 5c). Conversely, the expression levels of ICAM-1 and TNF$\alpha$ were significantly higher in the SHRcp animals than in the controls, and this expression was significantly attenuated with teneligliptin treatment (Figures 5d and e).

\section{DISCUSSION}

SHRcp rats exhibited obesity, hypertension, insulin resistance, glucose intolerance and endothelial dysfunction as they aged. Interestingly, long-term treatment with teneligliptin (for example, from 12 to 24 weeks of age) significantly improved endothelial dysfunction, suggesting that teneligliptin may have beneficial effects beyond that of lowering blood sugar. Consistent with the results from the SHRcp model, clinical trials involving patients with type 2 diabetic mellitus demonstrated that DPP-4 inhibitors attenuated endothelial dysfunction, as measured by the reactive hyperemia peripheral arterial tonometry index and flow-mediated dilatation. ${ }^{15-17}$ Sitagliptin (a DPP-4 inhibitor) enhanced Ach-induced endothelium-dependent vasodilation in aortic rings from apolipoprotein E-deficient mice. ${ }^{18}$ Furthermore, sitagliptin was shown to enhance the phosphorylation of eNOS through the cyclic adenosine monophosphate/protein kinase A pathway in human coronary artery endothelial cells by possibly augmenting the activity of glucagon-like peptide-1 on the endothelium. ${ }^{18}$ This effect could explain the effects on endothelial function of DPP-4 inhibitors that are beyond glucose control. Another explanation for these effects is anti-inflammatory activity. In a previous study, treatment with sitagliptin reduced the mRNA levels of several inflammatory mediators, including interleukin-6, monocyte chemoattractant protein-1 and TNF- $\alpha$, by significantly increasing cytosolic glucagon-like peptide-1-induced cyclic adenosine monophosphate levels, resulting in the inhibition of nuclear factor- $\mathrm{\kappa B}$ p65 nuclear translocation via the cyclic adenosine monophosphate/ protein kinase A pathway. ${ }^{18}$ Therefore, activation of the cyclic adenosine monophosphate/protein kinase A pathway may regulate inflammatory cytokine expression via an nuclear factor- $\mathrm{\kappa B}$-dependent pathway. Indeed, it was shown that sitagliptin treatment over a 6month period decreased high-sensitivity C-reactive protein levels in patients with type 2 diabetes mellitus. ${ }^{16}$ Consistent with previous reports, we also confirmed that teneligliptin treatment decreased TNF- $\alpha$ and ICAM- 1 expression and increased eNOS expression due, in part, to alleviating inflammation in the vasculature system.

A recent study elucidated the X-ray co-crystal structures of six inhibitors in complex with DPP-4, and these inhibitors were categorized into three classes based on their binding subsites: (class 1) vildagliptin and saxagluptin; (class 2) alogliptin and 

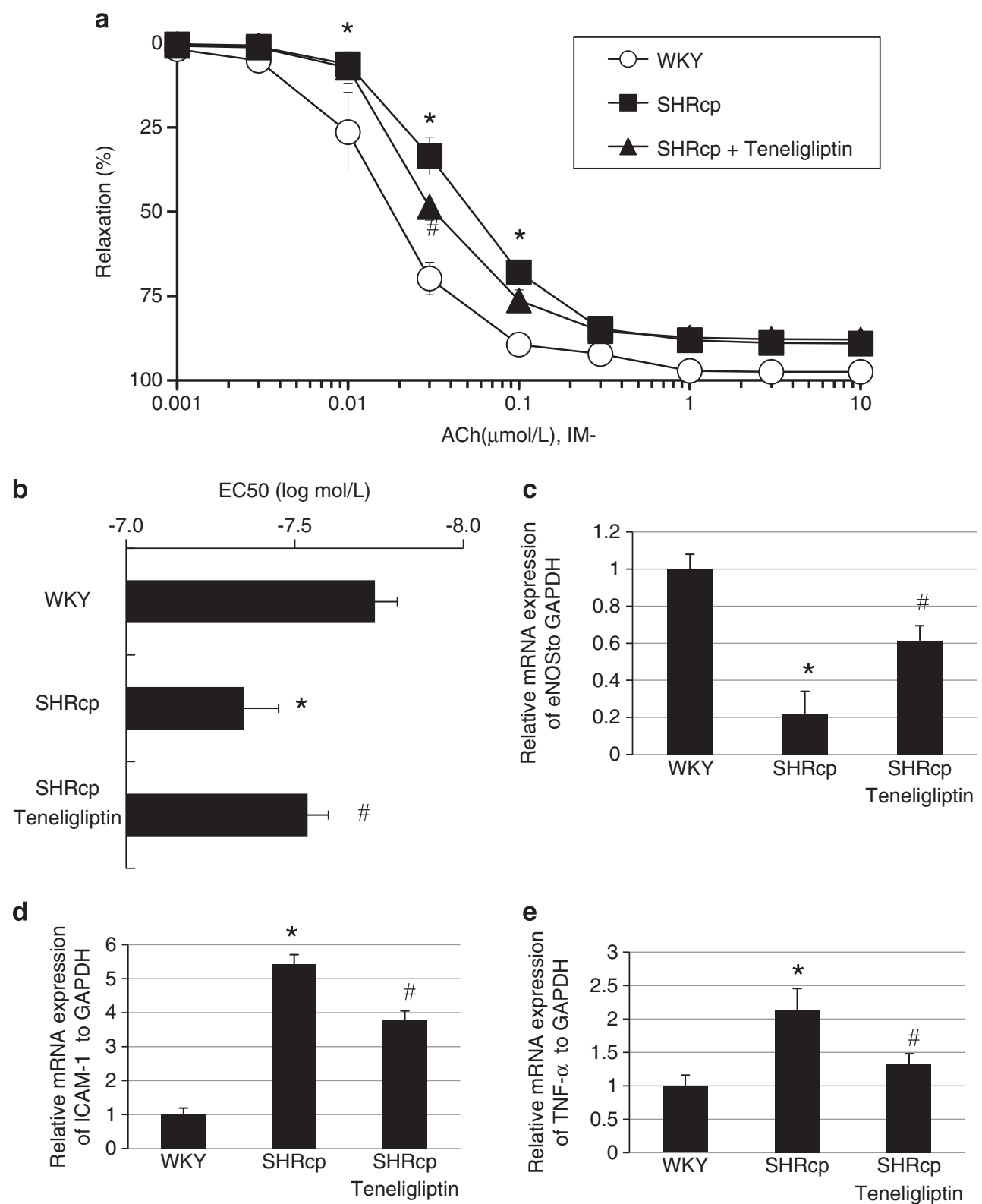

Figure 5 Endothelial function as assessed by the vasodilator response to acetylcholine (Ach).(a) Cumulative concentration-response curves for acetylcholine (Ach) treatment of isolated mesenteric arterial rings. Relaxation rates were calculated as a percentage of the maximum relaxation rate induced by papaverine. (b) Half maximal effective concentration ( $\mathrm{EC}_{50}$ ) values for acetylcholine (Ach)-induced relaxation calculated using nonlinear least squares regression analysis. (c-e) Expression levels of endothelium-derived nitric oxide synthase (eNOS), tumor necrosis factor (TNF)- $\alpha$ and intercellular adhesion molecule-1 (ICAM-1) mRNAs relative to glyceraldehyde-3-phosphate dehydrogenase (GAPDH) as determined using real-time reverse transcription-PCR (RTPCR). $N=3,6$ and 8 for each group, respectively. ${ }^{*} P<0.05$ vs. WKY rats, ${ }^{\#} P<0.05$ vs. SHRcp rats.

linagliptin; and (class 3) sitagliptin and teneligliptin. The existence of additional interactions with extensive subsites in class 3 DPP-4 inhibitors was also found, which may increase DPP-4 inhibition beyond the levels afforded by primary substrate interactions. ${ }^{19}$ On the basis of an evaluation of the inhibitory activity of six DPP-4 inhibitors, the $\mathrm{IC}_{50}$ value of teneligliptin $\left(1.9 \mathrm{nmoll}^{-1}\right)$ was found to be much lower than those of vidagliptin, alogliptin and saxagliptin $\left(29.2,4.9\right.$ and $6.3 \mathrm{nmoll}^{-1}$, respectively). ${ }^{20}$ In our present study, long-term treatment with teneligliptin significantly improved insulin resistance and hyperglycemia. Insulin resistance is partially caused by impaired insulin signaling within endothelial cells, and impaired insulin signaling (via decreased eNOS phosphorylation) in endothelial cells reduces insulin-induced glucose uptake. Thus, decreased insulin resistance caused by teneligliptin could also affect endothelial function. Interestingly, it has been reported that DPP-4 cleaves not only glucagon-like peptide-1 but also other peptides (for example, SDF- $1 \alpha$, neuropeptide $\mathrm{Y}$ and substance $\mathrm{P}$ ) that are known to have regulatory roles in vascular cells. ${ }^{21-23}$ Sitagliptin increases the number of endothelial progenitor cells in patients with type 2 diabetes mellitus, which may affect endothelial function through the modulation of SDF- $1 \alpha^{24}$ These combined effects of DPP-4 inhibitors may contribute to the improvement of endothelial function. 
In conclusion, long-term treatment with the DPP-4 inhibitor teneligliptin significantly improved endothelial dysfunction and glucose metabolism in a rat model of metabolic syndrome. From a clinical standpoint, this study suggests that teneligliptin treatment may help improve endothelial function in obese patients with severe hypertension and diabetes mellitus.

\section{CONFLICT OF INTEREST}

The authors declare no conflict of interest.

\section{ACKNOWLEDGEMENTS}

We thank Ms Hizuki Hamada and Ms Yoko Horiguchi for their technical assistance, and Ms Satoe Kitabata for her help in office procedures. This study was partially supported by Mitsubishi Tanabe. The Department of Clinical Gene Therapy was financially supported by Takeda, Daiichi-Sankyo, AnGes MG, Novartis, Shionogi, Boeringher and Rohto. The Division of Vascular Medicine and Epigenetics was financially supported by Baeyel and partially supported by Kowa and Astrazeneca.

1 Garrison RJ, Kannel WB, Stokes J 3rd, Castelli WP. Incidence and precursors of hypertension in young adults: the Framingham Offspring Study. Prev Med 1987; 16: 235-251.

2 Lind $\mathrm{L}$, Berne $\mathrm{C}$, Lithell $\mathrm{H}$. Prevalence of insulin resistance in essential hypertension. J Hypertens 1995; 13: 1457-1462.

3 Williams SB, Cusco JA, Roddy MA, Johnstone MT, Creager MA. Imparied nitric oxide-mediated vasodilation in patients with non-insulin-dependent diabetes mellitus. J Am Col Cardiol 1996; 27: 567-574.

4 Abbasi F, Asagmi T, Cooke JP, Lamendola C, McLaughlin T, Reaven GM, Stuehlinger M, Tsao PS. Plasma concentrations of asymmetric dimethylarginine are increased in patients with type 2 diabetes mellitus. Am J Cardiol 2001; 88: 1201-1203.

5 Chiu JD, Richey JM, Harrison LN, Zuniga E, Kolka CM, Kirkman E, Ellmerer M, Bergman RN. Direct administration of insulin into skeletal muscle reveals that the transport of insulin across the capillary endothelium limits the time course of insulin to activate glucose disposal. Diabetes 2008; 57: 828-835.

6 Barrett EJ, Eggleston EM, Inyard AC, Wang H, Li G, Chai W, Liu Z. The vascular actions of insulin control its delivery to muscle and regulate the rate-limiting step in skeletal muscle insulin action. Diabetologia 2009; 52: 752-764.

7 Kubota T, Kubota N, Kumagai H, Yamaguchi S, Kozono H, Takahashi T, Inoue M, Itoh S, Takamoto I, Sasako T, Kumagai K, Kawai T, Hashimoto S, Kobayashi T, Sato M, Tokuyama K, Nishimura S, Tsunodam M, Idem T, Murakami K, Yamazaki T, Ezaki O, Kawamura K, Masuda H, Moroi M, Sugi K, Oike Y, Shimokawa H, Yanagihara N, Tsutsui M, Terauchi Y, Tobe K, Nagai R, Kamata K, Inoue K, Kodama T, Ueki K, Kadowaki T. Impaired insulin signaling in endothelial cells reduces insulin-induced glucose uptake by skeletal muscle. Cell Metab 2011; 13: 294-307.

8 Holst JJ. Implementation of GLP-1 based therapy of type 2 diabetes mellitus using DPP-IV inhibitors. Adv Exp Med Biol 2003; 524: 263-279.

9 Drucker DJ. Therapeutic potential of dipeptidyl peptidase IV inhibitors for the treatment of type 2 diabetes. Expert Opin Investig Drugs 2003; 12: 87-100.
10 Takaya K, Ogawa Y, Hiraoka J, Hosoda K, Yamori Y, Nakao K, Koletsky RJ. Nonsense mutation of leptin receptor in the obese spontaneously hypertensive Koletsky rat. Nat Genet 1996; 14: 130-131.

11 Nagase M, Matsui H, Shibata S, Gotoda T, Fujita T. Salt-induced nephropathy in obese spontaneously hypertensive rats via paradoxical activation of the mineralocorticoid receptor: role of oxidative stress. Hypertension 2007; 50: 877-883.

12 Nagase M, Yoshida S, Shibata S, Nagase T, Gotoda T, Ando K, Fujita T. Enhanced aldosterone signaling in the early nephropathy of rats with metabolic syndrome: possible contribution of fat-derived factors. J Am Soc Nephrol 2006; 17 : 3438-3446.

13 Sueta D, Nakamura T, Dong YF, Kataoka K, Koibuchi N, Yamamoto E, Toyama K, Yasuda O, Ogawa H, Kim-Mitsuyama S. Amlodipine enhances amelioration of vascular insulin resistance, oxidative stress, and metabolic disorders by candesartan in metabolic syndrome rats. Am J Hypertens 2012; 25: 704-710.

14 Hayashi K, Nakamura S, Morishita R, Moriguchi A, Aoki M, Matsumoto K, Nakamura T, Kaneda Y, Sakai N, Ogihara T. In vivo transfer of human hepatocyte growth factor gene accelerates re-endothelialization and inhibits neointimal formation after balloon injury in rat model. Gene Ther 2000; 7: 1664-1671.

15 Van Poppel PC, Netea MG, Smits P, Tack CJ. Vidagliptin improves endothelium-dependent vasodilatation in type 2 diabetes. Diabetes Care 2011; 34: 2072-2077.

16 Matsubara J, Sugiyama S, Akiyama E, Iwashita S, Kurokawa H, Ohba K, Maeda H, Fujisue K, Yamamoto E, Kaikita K, Hokimoto S, Jinnouchi H, Ogawa H. Dipeptidyl peptidase-4 inhibitor, sitagliptin, improves endothelial dysfunction in association with its anti-inflammatory effects in patients with coronary artery disease and uncontrolled diabetes. Circ J 2013; 77: 1337-1344.

17 Kubota Y, Miyamoto M, Takagi G, Ikeda T, Kirinoki-Ichikawa S, Tanaka K, Mizuno K. The dipeptidyl peptidase-4 inhibitor sitagliptin improves vascular endothelial function in type 2 diabetes. J Korean Med Sci 2012; 27: 1364-1370.

18 Matsubara J, Sugiyama S, Sugamura K, Nakamura T, Fujiwara Y, Akiyama E, Kurokawa H, Nozaki T, Ohba K, Konishi M, Maeda H, Izumiya Y, Kaikita K, Sumida H, Jinnouchi H, Matsui K, Kim-Mitsuyama S, Takeya M, Ogawa H. A dipeptidyl peptidase-4 inhibitor, des-fluoro-sitagliptin, improves endothelial function and reduces atherosclerotic lesion formation in apolipoprotein E-deficient mice. J Am Coll Cardiol 2012; 59: 265-276.

19 Nabeno M, Akahoshi F, Kishida H, Miyaguchi I, Tanaka Y, Ishii S, Kadowaki T. A comparative study of the binding modes of recently launched dipeptidyl peptidase IV inhibitors in the active site. Biochem Biophys Res Commun 2013; 434: 191-196.

20 Kimata H, Fukuda-Tsuru S, Yoshida K, Iwasaki S, Kiuchi S, Akahoshi F, Ishii S. Teneligliptin, a novel selective dipeptidyl peptidase-4 inhibitor, improves postprandial hyperglycemia in a model of type 2 diabetes, zucker diabetic fatty rats. Med Consult New Remedies 2012; 49: 9.

21 Brown NJ, Byiers S, Carr D, Maldonado M, Warner BA. Dipeptidyl peptidase-IV inhibitor use associated with increased risk of ACE inhibitor-associated angioedema. Hypertension 2009; 54: 516-523.

22 Guan X, Karpen HE, Stephens J, Bukowski JT, Niu S, Zhang G, Stoll B, Finegold MJ, Holst JJ, Hadsell D, Nichols BL, Burrin DG. GLP-2 receptor localizes to enteric neurons and endocrine cells expressing vasoactive peptides and mediates increased blood flow. Gastroenterology 2006; 130: 150-164.

23 Huang CY, Shih CM, Tsao NW, Lin YW, Huang PH, Wu SC, Lee AW, Kao YT, Chang NC, Nakagami H, Morishita R, Ou KL, Hou WC, Lin CY, Shyu KG, Lin FY. Dipeptidyl peptidase-4 inhibitor improves neovascularization by increasing circulating endothelial progenitor cells. Br J Pharmacol 2012; 167: 1506-1519.

24 Fadini GP, Agostini C, Sartore S, Avogaro A. Endothelial progenitor cells in the natural history of atherosclerosis. Atherosclerosis 2007; 194: 46-54. 